\title{
Nonlinearities in the Real Exchange Rates: New Evidence from Developed and Developing Countries
}

\author{
By \\ Yamin Ahmad, Ming Chien Lo, and Olena Staveley-O’Carroll
}

December 2018

\section{COLLEGE OF THE HOLY CROSS, DEPARTMENT OF ECONOMICS \\ FACULTY RESEARCH SERIES, PAPER NO. 18-13*}

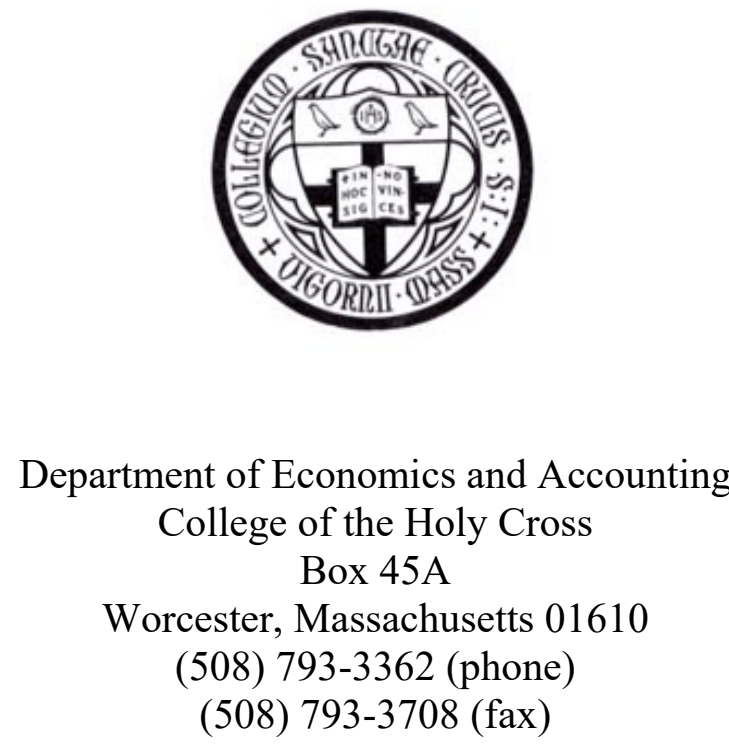

https://www.holycross.edu/academics/programs/economics-and-accounting

*All papers in the Holy Cross Working Paper Series should be considered draft versions subject to future revision. Comments and suggestions are welcome. 


\title{
Nonlinearities in the Real Exchange Rates: New Evidence from
}

\section{Developed and Developing Countries}

\author{
Yamin Ahmad* \\ Ming Chien $\mathrm{Lo}^{\dagger}$ \\ University of Wisconsin - Whitewater Metropolitan State University \\ Olena Staveley-O’Carroll ${ }^{\ddagger}$ \\ College of the Holy Cross
}

\begin{abstract}
This paper investigates nonlinearities in the dynamics of real exchange rates. We use Monte Carlo simulations to establish the size properties of the Teräsvirta-Anderson (1992) and the Teräsvirta (1994) test, when the dynamics of the real exchange rate is influenced by an exogenous process. In addition, we examine the modification proposed by Ahmad, Lo and Mykhaylova (2013; Journal of International Economics) to show that the modified nonlinearity test performs much better than the original in both Monte Carlo exercises and in the actual data on 1431 bilateral real exchange rate series. Finally, we investigate the dynamics of the real exchange rate for both developed and developing countries using the modified test for the recent floating period. In general, the results finds a greater incidence of nonlinear dynamics for developing country real exchange rates.
\end{abstract}

JEL Classification: F41 F47 C15 C32

Keywords: real exchange rate dynamics, nonlinear dynamics, smooth transition estimation, Monte Carlo analysis

Current Version: October 15, 2018

\footnotetext{
* Department of Economics, 800 W Main Street, Whitewater, WI 53190 Email: ahmady@uww.edu.

$\dagger$ College of Management, Metropolitan State University, 1501 Hennepin Avenue, Minneapolis, MN 55403-1897, Email: ming.lo@metrostate.edu

$\ddagger$ Corresponding author. Department of Economics and Accounting, College of the Holy Cross, 1 College Street, Worcester, MA 01610 USA. Email: omykhayl@holycross.edu. Phone: (508) 793-2736.
} 


\section{Introduction}

During the last decade, econometric models examining nonlinearities in the dynamics of real exchange rates have become popular among empirical researchers 1 However, since these models are rarely built on an explicit theoretical framework, their predictions need to be interpreted with great care. In fact, Ahmad, Lo, and Mykhaylova (2013), ALM hereafter, demonstrate that the ability of empirical tests to detect nonlinearities in real exchange rates (RERs) hinges critically on the underlying data generating process. Within a theoretical framework, the authors show that linearity tests - which express the real exchange rate as a univariate function of its own lags - suffer from misspecification in some cases, which consequently leads them to overestimate the incidence of nonlinearity in the RER series. In a follow-up to this finding, and with the aim of helping researchers and policymakers better understand the dynamics of real exchange rates, we now apply the ALM approach proposed in the original paper to empirical data. Additionally, we study the relationship between the data generating process that drives RERs and the results of standard nonlinearity tests.

The notion that real exchange rates possess nonlinear behavior became popular in the late 1990's due to the failures of a linear framework: assuming linear dynamics and perfect arbitrage implies that the speed of adjustment is constant at all levels of deviations from Purchasing Power Parity (PPP). However, the assumption of perfect arbitrage is not realistic, and nonlinearities in real exchange rates could exist due to, for example, transactions costs (Michael, Nobay and Peel, 1997; Obstfeld and Taylor, 1997), heterogeneity of agent's beliefs (Kilian and Taylor, 2003), and misalignments in the foreign exchange market resulting in a lack of co-ordination (Sarno and Taylor, 2001;

\footnotetext{
${ }^{1}$ For recent surveys on the literature which highlight the dynamics of real exchange rates characterized as a nonlinear process, see Taylor and Taylor (2004), Taylor (2006) and Ahmad and Glosser (2011). Engel, Mark and West (2007) provide a thorough overview of the developments in both theoretical and empirical literature.
} 
Reitz and Taylor, 2008). These papers are among many which have found that the data on real exchange rates can be parsimoniously characterized as smooth transition autoregressive (STAR) process, or one of its variants ${ }^{2}$ However, these studies lack rigorous theoretical foundations that could help to explain the existence of nonlinearities in RER behavior. Consequently, the tests of real exchange rate dynamics are based on ad-hoc univariate data generating processes, and their result can potentially be misleading due to misspecification problems.

We begin by re-examining the evidence of real exchange rate nonlinearity using the data on multiple bilateral rates during the most recent floating period (1970-2017). Of the 1431 bilateral RER series tested, the original Teräsvirta and Anderson (1992) test (TA test henceforth) found approximately 36 percent to be nonlinear. After we modify the test to include relative outputs, capital stocks, and inflations - the variables found by ALM to significantly improve test performance - close to a fifth of all the series are re-classified (both from initially linear to now nonlinear, and vice versa). The patterns in the data also suggest that the real exchange rates of developing countries have a higher incidence of nonlinearity than those of advanced economies.

To better understand the causes of switches between linear and nonlinear classification under the TA and ALM tests, we next turn to Monte Carlo experiments, which allow us to control the RER data generating process. Of course, there are infinitely many possible processes that can be used to characterize the dynamics of the real exchange rate. In this paper, we restrict our attention to the case in which the real exchange rate depends linearly on its lags and lags of the exogenous variables. We vary the parameters that govern the persistence of this process and the variance of white noise shocks, and examine the impact of these variations on the TA and ALM test outcomes. Our results indicate that (a) the TA test often misinterprets the presence of exogenous variables that drive

\footnotetext{
${ }^{2}$ A small sample of papers include Sarantis (1999), Baum et al (2001), Taylor et al (2001), and Paya and Peel (2006).
} 
RERs as nonlinearities, confirming the theoretical findings in Ahmad, Lo and Mykhaylova (2013); and (b) the incidence of detected nonlinearity increases with the persistence of exogenous variables. In contrast, the augmented ALM test fares much better: the rate of rejection of the null hypothesis (of a linear process) is approximately equal to the size of the test for all parameter values under consideration. Observation (a) above parallels our earlier empirical findings: about a quarter of the real exchange rates that were initially characterized as nonlinear STAR processes are found to be linear under the ALM modification. Thus, we would like to extend a word of caution to empirical researchers: without a theoretical model of real exchange rate behavior, they risk overestimating the incidence of nonlinearities in its dynamics due to the influence of exogenous variables.

The remainder of the paper is structured as follows: the standard nonlinearity test and its potential misspecification are outlined in Section 2. Section 3 discusses the test modification proposed in ALM and compares the results of the two tests using bilateral RER data for the 1970-2017 period. Section 4 employs a series of Monte Carlo exercises to better understand the relationship between test performance and the underlying data generating process and to primarily study the size properties of the TA and ALM tests. Finally, Section 5 concludes and highlights the avenues for future research.

\section{Testing for Nonlinearities in the RER Series}

\subsection{Tests of RER nonlinearity}

Ahmad, Lo and Mykhaylova (2013) demonstrate that time series tests of nonlinearities present in the real exchange rate dynamics may be misspecified due to the omission of exogenous variables responsible for RER dynamics. We refer the interested reader to their paper for a detailed descrip- 
tion of the misspecification problem and the proposed solution to it; below, we briefly outline its main findings.

In general, empirical tests assume that the log real exchange rate $q_{t}$ may be succinctly captured by a univariate autoregressive process of the form

$$
q_{t}=\lambda_{1} q_{t-1}+\ldots+\lambda_{p} q_{t-p}+\varepsilon_{t}
$$

where $\varepsilon_{t}$ is white noise. The RER series is stationary if the sum of the autoregressive coefficients, $\Lambda \equiv\left|\sum_{i=1}^{p} \lambda_{i}\right|$, is less than 1.

Several recent papers have argued that a possible resolution to Rogoff's (1996) PPP puzzle is to model the real exchange rate as a nonlinear stationary process. The nonlinearities have taken the form of Markov switching, threshold processes, and variants of smooth transition (STAR) models. Although the nonlinear modeling strategies differ across papers, all such studies aim to show that small permanent deviations from PPP are possible due to frictions, whereas large deviations are quickly corrected. Consequently, real exchange rates exhibit mean-reverting behavior only when there is a substantial deviation from the level implied by purchasing power parity.

As a first step to understanding the dynamics of the real exchange rates, we limit our attention to the STAR type nonlinearity, given its popularity and success in the literature. The dynamics of the real exchange rate (which can also be thought of as a deviation from PPP) can be described as a STAR process as follows:

$$
q_{t}=\sum_{j=1}^{p} \lambda_{j} q_{t-j}+\left(\sum_{j=1}^{p} \tilde{\lambda}_{j} q_{t-j}\right) F\left(q_{t-d}, \kappa\right)+\varepsilon_{t},
$$

where $\left\{q_{t}\right\}$ is a globally stationary ergodic process, $\varepsilon_{t} \sim i i d W N\left(0, \sigma^{2}\right)$, and $F($.$) represents a$ 
transition function from one regime to another and determines the degree of mean reversion. Finally, $d$ and $\kappa$ represent the delay and speed of adjustment parameters, respectively. According to (2), the dynamics of the real exchange rate are driven by the autoregressive parameters $\left\{\lambda_{1}, \ldots, \lambda_{p}\right\}$ in one regime, and $\left\{\lambda_{1}+\tilde{\lambda}_{1}, \ldots, \lambda_{p}+\tilde{\lambda}_{p}\right\}$ in the other. The popularity of the STAR framework arises from its ability to demonstrate how the real exchange rate may move smoothly from one type of regime to another, depending on how far the RER value is from a particular threshold. Small deviations from PPP are considered by the STAR framework to be persistent, whereas large deviations exhibit mean-reverting dynamics. Generally, it is assumed that nonlinearity is present if the transition function in (2) does not equal zero; this test can be captured by the null hypothesis $H_{0}: \kappa=0$.

We outline the basic elements of the linearity test, given its relevance for the question being asked in our paper, in the next section.

\subsection{DGP and Test Misspecification}

We now examine the correspondence between the data generating process (DGP) of the simulated RER series and the specifications (1) and (2). The simulated data comes from an n-th order Taylor expansion around the steady state of the theoretical DSGE model described in ALM. The solution approximated to the first order expresses the current value of the endogenous variables as a function of the previous state of the model and the realization of shocks at the beginning of the current period:

$$
\begin{aligned}
& \mathbf{u}_{t}=A_{u}+B_{u} \mathbf{x}_{t-1}+C_{u} \varepsilon_{t} \\
& \mathbf{x}_{t}=A_{x}+B_{x} \mathbf{x}_{t-1}+C_{x} \varepsilon_{t}
\end{aligned}
$$


where $\mathbf{u}_{t}$ is a $k_{u} \times 1$ vector of non-predetermined variables (controls); $\mathbf{x}_{t}$ is a $k_{x} \times 1$ vector of predetermined endogenous variables (states); $\varepsilon_{t}$ is a $k_{\varepsilon} \times 1$ vector of predetermined exogenous variables (shocks); and $A_{i}, B_{i}$ and $C_{i}, i \in\{u, x\}$ are appropriately-dimensioned coefficient matrices.

Given that the focus of our paper is on the real exchange rate, assume, for illustrative purposes and without loss of generality, that $k_{u}=1$ so that $u_{t}=q_{t}$. ALM show that the RER series generated by the process (3) cannot be re-written as a univariate $A R(p)$ equation (1), except for the simple case of $k_{x}=1$. Instead, if we were to express $q_{t}$ as a function of its own lags, we would instead obtain an ARMAX process of the following form:

$$
q_{t}=\phi_{0}+\sum_{j=1}^{p} \phi_{j} q_{t-j}+\varphi_{x}^{\prime} \mathbf{x}_{t-2}+\varphi_{\varepsilon}^{\prime} \varepsilon_{t-1}+C_{u} \varepsilon_{t}
$$

where $\varphi_{x}$ and $\varphi_{\varepsilon}$ are functions of the coefficients of $A_{i}, B_{i}, C_{i}, i \in\{u, x\}$ and of the sum of the autoregressive coefficients, $\sum_{j=1}^{p} \phi_{j}$. Hence, failure to account for the presence of the lagged state variables on the right hand side of (4) when estimating the DGP would lead to an omitted variables problem. Consequently, estimates of the autoregressive coefficients used to represent the dynamics of the real exchange rate may be biased. Conceivably, a parsimonious nonlinear process may capture the dynamics of the data quite well in the presence of omitted variables under a linear approximation to the model's equations. A simple approach to resolve this issue would be to include some subset of relevant lagged state variables in the nonlinearity test. We would then expect to see a reduction in the incidence of nonlinearity down to the size (plus any size distortions) of the test.

Second-order approximation to the model's equations introduces nonlinearities into the dynamics 
of endogenous variables as follows:

$$
\begin{aligned}
& \mathbf{u}_{t}=A_{u}+B_{u} \mathbf{x}_{t-1}+C_{u} \varepsilon_{t}+D_{u}\left(\mathbf{x}_{t-1} \otimes \mathbf{x}_{t-1}\right)+E_{u}\left(\varepsilon_{t} \otimes \varepsilon_{t}\right)+G_{u}\left(\mathbf{x}_{t-1} \otimes \varepsilon_{t}\right) \\
& \mathbf{x}_{t}=A_{x}+B_{x} \mathbf{x}_{t-1}+C_{x} \varepsilon_{t}+D_{x}\left(\mathbf{x}_{t-1} \otimes \mathbf{x}_{t-1}\right)+E_{x}\left(\varepsilon_{t} \otimes \varepsilon_{t}\right)+G_{x}\left(\mathbf{x}_{t-1} \otimes \varepsilon_{t}\right)
\end{aligned}
$$

Suppose again that $\mathbf{u}_{t}=q_{t}$. If we were to estimate the equation for $q_{t}$ as an autoregressive process including only the linear terms:

$$
q_{t}=\phi_{0}+\sum_{j=1}^{p} \phi_{j} q_{t-j}+\varphi_{x}^{\prime} \mathbf{x}_{t-2}+\varphi_{\varepsilon}^{\prime} \varepsilon_{t-1}+C_{u} \varepsilon_{t}
$$

then incidences of nonlinearity beyond the size of the test would indicate the presence of the higher order terms in the RER DGP. Indeed, ALM find that the two-country DSGE models featuring incomplete international asset markets and nontraded goods generate nonlinearities in the simulated RER data that can be identified by time series methods. The paper also suggests that the state variables $\mathbf{x}_{t}$ necessary to correct the omitted variables problem include both countries' outputs, capital stocks, and consumer price inflations.

In the empirical section of the paper, we test these findings by applying them to the actual data on real exchange rates, output, inflation, and capital stock.

\section{New evidence from empirical exercises}

\subsection{Testing methodology}

We begin our empirical analysis by applying the findings of ALM to a large number of bilateral real exchange rates during the 1970-2017 period; see Appendix A for data sources and description. We 
subject each series to two nonlinearity tests: the original Teräsvirta and Anderson (1992) test, and the ALM modification outlined below. We briefly describe the details of each test next, although we refer the reader to Teräsvirta (1994) or Teräsvirta and Anderson (1992) for additional details regarding the overall estimation methodology.

Empirical estimation of STAR models, described in (2), proceeds in three steps. Once the order of autoregression, $p$, has been determined through traditional means (e.g., utilizing information criteria like the AIC/SBC), these steps include: testing for linearity; selecting the value of the delay parameter, $d$; and choosing between LSTAR (logistic smooth transition) and ESTAR (exponential smooth transition) specifications. These differ in their assumptions about the functional form of the transition function $F(\bullet)$ in (2); the former postulates an asymmetric RER adjustment process, whereas the latter assumes that real exchange rates adjust symmetrically to both positive and negative deviations from PPP.

The RER series is presumed to be linear if the parameter $\kappa$ in $(2)$ is equal to zero. If $\kappa$ were known, then it would be possible to proceed using classical inference techniques. However, since $\kappa$ and $d$ are typically not known in practice, then (2) is not identified under the null hypothesis, and hence no consistent estimate of either $\kappa$ or $d$ exists. This is the essence of the problem outlined by Davies (1977). To address this issue, Teräsvirta (1994) follows the suggestion proposed in Davies (1977) and keeps the unidentified values fixed when deriving a Lagrange Multiplier (LM)-type test for linearity. The idea behind the test statistic used to test $H_{0}: \kappa=0$ involves taking a third order Taylor expansion of the transition function (2) around $\kappa=0$. Taking $p$ as given, the researcher estimates the auxiliary regression below for a fixed parameter $d$ :

$$
q_{t}=\alpha_{00}+\sum_{j=1}^{p}\left(\alpha_{0 j} q_{t-j}+\alpha_{1 j} q_{t-j} q_{t-d}+\alpha_{2 j} q_{t-j} q_{t-d}^{2}+\alpha_{3 j} q_{t-j} q_{t-d}^{3}\right)+\varepsilon_{t}
$$


and tests the joint null hypothesis that all the coefficients corresponding to the cross products in (6) are zero:

$$
H_{0, L I N}=\alpha_{1 j}=\alpha_{2 j}=\alpha_{3 j}=0 \quad(j=1, \ldots, p)
$$

Nonlinearity is detected if the researcher is able to reject $H_{0, L I N}$. The test above is also used to determine the delay length, $d$, by running the linearity test for all plausible values of $d$ and picking the one that minimizes the test's p-value (as suggested by Tsay, 1989).

However, given that the RER data generating process may involve several other variables, the test in (6) may be misspecified. Therefore, we augment it by including three additional lagged variables: output, capital stock, and inflation. As discussed in ALM, this choice of state variables is influenced by the ease of obtaining their real-world equivalents. Output and capital stocks capture and summarize developments in the real side of the economy; the third state variable is meant to control for inflation persistenç3 The modified test takes the following form:

$$
\begin{aligned}
q_{t}= & \alpha_{00}+\sum_{j=1}^{p}\left(\alpha_{0 j} q_{t-j}+\alpha_{1 j} q_{t-j} q_{t-d}+\alpha_{2 j} q_{t-j} q_{t-d}^{2}+\alpha_{3 j} q_{t-j} q_{t-d}^{3}\right) \\
& \beta_{y} y_{t-1}+\beta_{k} k_{t-1}+\beta_{\pi} \pi_{t-1}+\varepsilon_{t},
\end{aligned}
$$

with the null hypothesis still given by (7). Appendix A presents a brief description of the construction of the capital stock series; detailed calculations are available from the authors upon request.

\subsection{Real exchange rates around the world}

Tables 1 and 2 present the results of the empirical analysis. The typical approach in the literature is to conduct unit root tests prior to testing for nonlinearity in the real exchange rate series. We follow

\footnotetext{
${ }^{3}$ The choice of these particular right-hand-side variables is further addressed in the next section.
} 
the same approach here, although we do not use the results of these tests as a basis for determining the existence of nonlinearity, particularly given the well-known power properties of unit root tests in the time series literature $4^{4}$ Of the 1431 studied series, 510 are found to be nonlinear using the TA test in Table 1. This number decreases to 501 using the ALM modification. In both the TA and ALM cases, more than half of the series found to be nonlinear have the LSTAR specification, indicating asymmetric adjustment. However, the aggregate numbers mask the differences between the results of the two approaches.

In panel A of Table 2, we note that approximately eighty four percent of the series are found to have the same type of (linear or nonlinear) behavior using either of the two tests; the other sixteen percent of the series switch their type following the addition of the exogenous variables using the ALM test. Taking a closer look at these switches, we note that about a quarter of the RER series originally indicated to be a STAR process become linear, potentially indicating the presence of spurious nonlinearity. At the same time, the results of the ALM test indicate that about a tenth of the series which were initially linear according to the TA test are now described as a STAR process. In other words, the ALM modification leads to changes in the composition of both sets of exchange rates initially classified as linear or nonlinear. We interpret switches from models that were initially identified as STAR processes under the TA test to a linear process as confirmation of the proposition in Ahmad, Lo and Mykhaylova (2013) relating to the size of the TA test. They indicate evidence of misspecification. On the other hand, the presence of switches from linear to STAR models have to do with the power of the test. These types of switches may indicate the presence of true nonlinearities. In the next section, we employ a series of Monte Carlo exercises to

\footnotetext{
${ }^{4}$ Of the 1431 bilateral exchange rate series examined, 150 (approximately 10\%) reject the null hypothesis of a unit root. Although the low power properties of unit root tests are well documented in the literature, many studies reject the hypothesis of a random walk in the RER process in favor of persistent deviations from PPP that range from three to five years (see, among others, Chen and Engel, 2005). This is one of the reasons why the literature has adopted an approach to estimate STAR processes that may contain regimes that are nonstationary, but are globally stationary.
} 
better understand the conditions under which the TA and ALM tests reverse each other's findings. However, it may be instructive to first look for additional patterns in the RER data that may shed additional light on the properties of the two tests.

The results so far have been conducted for the full sample of countries. However, there is strong reason to suspect (based on evidence presented in several recent studies briefly summarized below) that the real exchange rate dynamics may be different between developed and developing economies, a conjecture we investigate next. Generally speaking, the difference can be driven by variations in the shock processes of the two groups of countries and/or by their asymmetric economic structure which translates into distinct transmission mechanisms of similar underlying shocks.

In many two-country theoretical models, the optimizing behavior of households equates the real exchange rate to the ratio of the home and foreign marginal utilities of consumption. Therefore, insofar as consumption is closely related to the dynamics of output, the TFP shock processes in the two countries significantly influence the real exchange rate behavior. Aguiar and Gopinath (2007) argue that the technology process of developing economies is characterized by permanent shocks, in contrast to fluctuations around the trend in the developed world. The implication of this finding (not pursued in the original paper) is that, via the consequently higher volatility of output and consumption, developing countries' RERs are more volatile and persistent compared to those of the advanced economies. This prediction is supported by the empirical evidence in Duarte et al. (2007) and Hausmann et al. (2006).

On the other hand, many empirical papers, including the seminal paper by Backus and Smith (1993), have challenged the RER-relative marginal utilities nexus, instead noting that the correlation between real exchange rates and relative consumptions is very low and often negative. This is quite likely the result of multiple real-world frictions - often absent from theoretical models - that 
are responsible for altering the relationship between the real exchange rate and the fundamentals. For example, Elbadawi and Soto (2008) note that taxes, government spending, and foreign aid are statistically significantly correlated with the real exchange rate in developing but not in OECD economies. Ganguly and Breuer (2010) suggest that currencies of developed economies trade in large and liquid foreign exchange markets, where the availability of various hedging instruments prevents large swings in exchange rate movements. Additionally, based solely on the decomposition of the real exchange rate into its nominal exchange rate and relative prices components and thus not subscribing to any underlying theoretical frameworks, the authors identify higher relative price volatility as the main cause of more volatile RERs in the developing countries. Higher relative inflation volatility in the developing world can, in turn, be ascribed to unstable economic and political institutions in the developing economies (Aisen and Veiga, 2006). Alternatively, Ahmad and Staveley-O'Carroll (2017) suggest that the decisions of exporting firms as to the choice of invoicing currency and the frequency of price re-optimization - which vary significantly depending on the destination markets - can explain a large portion of this difference. It is highly plausible that the differences in the inflation processes consequently affect the dynamics of RERs in different country subsamples.

The two explanations - persistent versus temporary productivity shocks and the role of price movements - implicitly support our choice of the controls included in the nonlinearity tests. While it is also reasonable to conjecture that the extent of international trade might affect RER dynamics of a country, both Duarte et al. (2007) and Hausmann et al. (2006) find that the degree of trade openness does not impinge on the resulting exchange rate moments.

We use the country classification from the IMF's World Economic Outlook 2012 to group countries into developing and developed categories ${ }^{5}$ We also consider the group of 17 Eurozone countries

\footnotetext{
${ }^{5}$ To ensure that the findings are robust, we recalculate the averages of the two moments of inflation using the
} 
(listed in Appendix A, motivated by the observation that fixing the nominal exchange rates may alter the dynamics of the bilateral RERs in the monetary union. The findings, presented in tables 2 and 3, reveal three broad trends that are robust to different country classification schemes.

1. The RERs of developing economies have a lower incidence of linearity (around 48 versus 64 percent for the advanced economies).

2. The frequency of switches from linear to nonlinear (in 11-17 percent of the series), depending on whether the TA or the ALM test is used, appears to be similar in all three subsamplesdeveloped, developing, or developed-developing pairs. However, the frequency of switches from nonlinear to linear is higher for developed economies and the Eurozone group (35 and 41 percent, respectively) than for developing (16 percent) and non-Eurozone (19 percent) countries.

3. The proportion of STAR models that switch to linear is higher than the proportion of linear models that switch to STAR specifications.

The second and third points above relate directly to the central thesis of this study, namely, whether the inclusion of the aforementioned exogenous variables helps to identify the appropriate reduced form model derived from the true structural model. Given that the largest portion of switches are from STAR to linear, the failure to include exogenous forcing variables may lead researchers to incorrectly detect the presence of nonlinear dynamics in RER series. This is true regardless of the level of development of the country. At the same time, noting that $11 \%$ to $17 \%$ of the series switch from linear to STAR dynamics, we can postulate that these real exchange rates may be truly

country classification provided by the World Bank World Development Report (we classify high income countries as developed and the rest as developing). Additionally, we also change the date of both classifications to 1992, coinciding with the middle of our sample. The results, which remain virtually unchanged, are available from the authors upon request. 
nonlinear (conditional on the set of exogenous variables appropriately characterizing the reduced form model).

Panel B of Table 2 provides further details on the breakdown of the switches under the TA and ALM tests. There we note that the majority of switches from linear to STAR dynamics indicate the data generating process is best characterized as an LSTAR process with asymmetric adjustment dynamics, more so for developing countries than developed countries.

Turning finally to the Eurozone economies (table 3), we find that the RER dynamics in this group of 17 economies is relatively more linear than in the non-Eurozone countries, and a relatively high fraction of nonlinearities detected with the TA test disappears once the we apply the ALM modification. For a plausible explanation of this pattern we can turn back to the original motivations, outlined in the introduction, for using nonlinear tests of RER dynamics: transaction costs associated with nominal currency conversions, heterogeneity of agents' beliefs, and lack of policy coordination. Arguably, the process of European integration that culminated in the creation of the common currency area smoothed out such frictions (and perhaps even eliminated some of them). As a result, the inner (nonlinear) regime captured in (2) is much smaller, and the RER dynamics is primarily captured by the outer (linear) regime, especially once the three exogenous indicators are added to the empirical test specification.

Based on these findings and the brief overview of the developed/developing RER properties presented above, we now take a closer look at the relationship between the RER series persistence and volatility and the results of the TA and ALM tests. 


\section{Uncovering the differences: Monte Carlo exercises}

In order to further investigate the relationship between the TA and ALM tests and the real exchange rate DGP, we turn to Monte Carlo simulations that allow us to control the RER data generating process without taking a stand on a particular theoretical model. In doing so, we wish to establish the size of the test in the presence of exogenous forcing variables that may indeed drive real exchange rate movements.

In reality, there are of course infinitely many possible DGPs that can be used to characterize the data, and these could be linear or nonlinear processes. The Box-Jenkins approach advocates the use of a parsimonious representation of the underlying data generating process. In order for us to establish the size properties of the linearity test, we begin by focusing on the subset of DGPs that are linear, under the assumption that RER dynamics are determined as linear functions of exogenous state variables. Even here, when we restrict our attention to this case, the underlying data generating process for the real exchange rate may (for example): (a) depend on its lags and lags of exogenous variables; or (b) be a function solely of lags of exogenous variables; or (c) be determined jointly with other variables through a vector autoregressive (VAR) process. Below, for simplicity, we focus on specification (a) by assuming that the real exchange rate $q_{t}$ and the forcing variable (for example output) $y_{t}$ evolve according to

$$
\begin{aligned}
q_{t} & =\alpha_{q}+\sum_{i=1}^{p} \beta_{q, i} q_{t-i}+\sum_{i=1}^{h} \beta_{q y, i} y_{t-i}+\varepsilon_{q, t} \\
y_{t} & =\alpha_{y}+\sum_{i=1}^{k} \beta_{y, i} y_{t-i}+\tau_{q y} \varepsilon_{y, t}
\end{aligned}
$$

The parameters governing the process in (9) can either be estimated from the data, or take on 
several values in a pre-specified range, the latter approach being more computationally costly. In our first experiment, we allow the parameters $\beta_{q}, \beta_{y}$, and $\tau_{q y}$ to take on several values; for tractability, we restrict the values of $p, h$, and $k$ to equal one, assume that both error terms $\varepsilon_{q, t}$ and $\varepsilon_{y, t}$ are independent white noise processes, and set the constant terms $\alpha_{q}$ and $\alpha_{y}$ equal to zero. More specifically, $\beta_{q, 1}$ and $\beta_{y, 1}$ take on values between 0.10 and 0.95 with the grid step of 0.05 ; the scaling parameter $\tau_{q y}$ ranges from 0.5 to 5 in increments of 0.5 . Finally, we (arbitrarily) set $\beta_{q y, 1}=0.375$. Thus, we consider a total of 2,890 sets of different parametric values. For each set, we simulate 10,000 real exchange rate series, each 450 observations long, and test the simulated data using the original Teräsvirta and Anderson (1992) test (6) as well as the ALM modification (8). In both tests, we set $d=1$.

Because our assumed DGP is linear, the null hypothesis (7) is correct. As long as there are no size distortions, the percentage of rejection of the null hypothesis, at a five percent significance level, should be 5\%. The results of the tests are shown in Figure 1. The horizontal axis captures the changes in the three parameter values. We begin by setting $\beta_{q, 1}=\beta_{y, 1}=0.1$ and let $\tau_{q y}$ range from 0.5 to 5 . Next, we gradually increase the value of $\beta_{q, 1}$ to 0.95 , iterating on the values of $\tau_{q y}$ for each incremental change in $\beta_{q, 1}$. Finally, we vary the parameter $\beta_{y, 1}$, iterating on $\beta_{q, 1}$ and $\tau_{q y}$ for each incremental increase. The top panel plots the null hypothesis rejection rates for each of the 2,890 parameter value sets using the TA test; the resulting pattern is repetitive but increasingly volatile. Two of the three parameters seem to be driving these results. First, as $\tau_{q y}$ increases from 0.5 to 5 , the number of rejections tends to fall, making the test more conservative in rejecting the null hypothesis. Second, for larger values of $\beta_{y, 1}$ (in other words, for more persistent dynamics of output), the rate of rejections increases, eventually exceeding $13 \%$ for $\beta_{y, 1}=0.95$.

When we do not include the right-hand side variable $y_{t}$ in the nonlinearity test, the estimated 

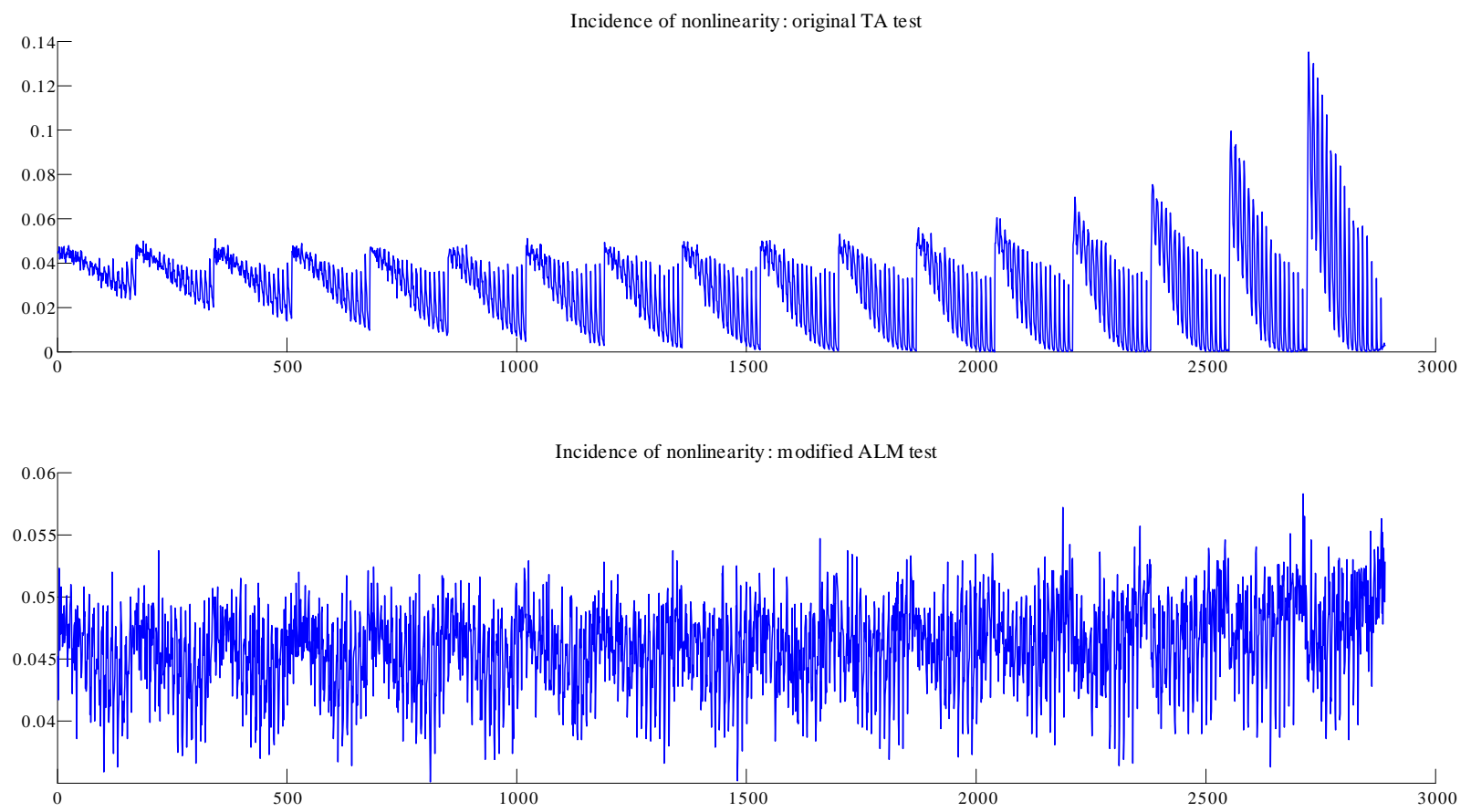

Figure 1: Estimated incidence of nonlinearity in the process $(9)$ using the original and the modified tests. Along the x-axis, $\beta_{y, 1}$ ranges from 0.10 to 0.95 ; for each value of $\beta_{y, 1}, \beta_{q, 1}$ increases from 0.10 to 0.95 in steps of 0.05 ; finally, for each increase in $\beta_{q, 1}$, we let the parameter $\tau_{q y}$ range from 0.5 to 5 .

variance of $\varepsilon_{y}$ is larger than its actual value. As the value of $\tau_{q y}$ increases, this estimate becomes larger still. As a result of the increased level of noise in the estimation, the TA test is less likely to reject the null hypothesis (7), since large standard errors suggest that the parameters $\alpha$ are insignificantly different from zero. Consequently, it is not surprising that the original test is more conservative in rejecting the null as $\tau_{q y}$ increases from 0.5 to 5 .

As expected, by including the exogenous variable $y_{t}$, the augmented ALM test fares better, as shown in the bottom panel of Figure 1. While small distortions are present, the null rejections rates for all parameter values under consideration lie within the $0.04-0.06$ range, very close to the size of the test.

To get a sense of the linearity tests' performance with longer lag lengths, in the second exercise we 
set $p=h=2$, and run the tests for $d=1,2$. We modify the parameters in $(9)$ as follows:

$$
\begin{aligned}
& q_{t}=1.2 q_{t-1}-0.35 q_{t-2}+0.4 y_{t-1}+0.35 y_{t-2}+\varepsilon_{q, t} \\
& y_{t}=1.1 y_{t-1}-0.2 y_{t-2}+2 \varepsilon_{y, t}
\end{aligned}
$$

Since the sum of the autoregressive parameters for both $q_{t}$ and $y_{t}$ (equal to 0.85 and 0.9 , respectively) is less than unity, both processes are stationary.

The results of nonlinearity tests, based on 10,000 simulations, each 450 periods long, are shown in the top panel of Table 4. To avoid complications, the values of the parameters $p$ and $h$ are the same in the tests and in the DGP. Just as in the previous exercise, the original TA test tends to over-estimate the incidence of nonlinearity of RER dynamics above the $5 \%$ size of the test, whereas the null rejection rate of the augmented ALM test is fairly close to 5\%. The value of the delay parameter $d$ does not seem to affect the results of either test.

Finally, to make sure that the augmented test does not over-parameterize the regression, we perform the same Monte Carlo simulation as in the exercise above with the DGP given by (10), except we now set $\beta_{q, 1}=\beta_{q, 2}=0$, thereby making the $q_{t}$ and $y_{t}$ series independent from one another. Notice that the real exchange rate dynamics are now governed by a purely autoregressive linear process. As indicated in the bottom panel of Table 4, the results of the augmented test are similar to that of the original test, although both are slightly conservative in rejecting the null hypothesis at the $5 \%$ confidence level.

We are therefore able to conclude that the augmented ALM test performs equally well or better than the original TA test when the real exchange rate is driven by exogenous variables in addition to its own lags. 
Having described the size characteristics of the two tests, it would make sense to examine the power properties of the test. However, here there are some issues that deserve a mention.

In examining the power of these tests, ideally, we would like to determine whether the ALM modification has equal or superior power over the TA approach when the alternative hypothesis is true. However, the Monte Carlo exercises that can be used to compare the power of the ALM and TA tests are complicated for three reasons. First, it is obvious that when the null hypothesis is rejected, there exists a potentially infinite number of alternatives. Following the trends in recent literature, we limit our choices to the STAR family (more specifically, the ESTAR and LSTAR specifications described in Section 3 above). Second, the regressions (6) and (8) used in the tests are approximations of the true STAR processes, and as such they in and of themselves could be the sources of low power due to misspecification of the true DGP. What leads us not to pursue the exploration of the power properties in this paper is the third reason: when we tested a few simulated time series - using the true nonlinear DGPs

$$
q_{t}=\sum_{j=1}^{p} \lambda_{j} q_{t-j}+\left(\sum_{j=1}^{p} \tilde{\lambda}_{j} q_{t-j}\right) F\left(q_{t-d}, \kappa\right)+\varepsilon_{q, t}
$$

and

$$
\begin{aligned}
q_{t} & =\sum_{j=1}^{p} \lambda_{j} q_{t-j}+\left(\sum_{j=1}^{p} \tilde{\lambda}_{j} q_{t-j}\right) F\left(q_{t-d}, \kappa\right)+\sum_{i=1}^{h} \beta_{q y, i} y_{t-i}+\varepsilon_{q, t} \\
y_{t} & =\alpha_{y}+\sum_{i=1}^{k} \beta_{y, i} y_{t-i}+\tau_{q y} \varepsilon_{y, t}
\end{aligned}
$$

rather than their approximated versions (6) and (8) to avoid the misspecification bias-both tests still appeared to suffer from severe lack of power, and we did not find any appreciable difference in their respective power. As Lo (2008) shows, a nonlinear DGP under certain STAR specifications can 
be easily mistaken for a very persistent but linear process. In particular, under certain assumptions the linear term $\alpha_{0 j} q_{t-j}$ may not be distinguishable from the other nonlinear terms in (6) and (8) in a Monte Carlo exercise. We suspect that the value of $d$ in the threshold variable $q_{t-d}$ could play a role in causing our results to have low power and in producing virtually no difference in the power of the test across different DGPs. Based on these observations, we believe that the exploration of the power properties of the two tests deserves to be addressed more thoroughly and extensively in separate future research.

\subsection{Back to the empirical patterns}

Developing and developed countries differ in the levels of RER volatility and persistence, likely due to differences in the TFP processes, international borrowing and lending, monetary policy and its resulting price dynamics. These hypothesized differences in the real exchange rate DGPs manifest themselves in higher levels of nonlinearity in the subsample of developing countries, and a higher incidence of switching from linear to STAR type dynamics, relative to those in developed countries. Given the results of the Monte Carlo analysis above, which indicate that the ALM modification performs better than the TA test in terms of the test size, we can conclude that these series should be characterized as truly nonlinear processes. However, given that this study is reduced form in nature, we are unable to uncover the specific types of shocks or structural features that characterize the economies of developing countries and cause the real exchange rates to mean-revert only following substantial deviations from a threshold level captured by the STAR framework. For example, the existence of trade barriers can result in the lack of movement in the real exchange rate as long as it remains relatively near the PPP-implied level. Testing this hypothesis within our framework would require including inter-country trade data as explanatory variables in the ALM 
test, an undertaking that we choose to leave to future work.

Real exchange rate dynamics between developed country pairs switch from STAR to linear processes under ALM in a higher proportion as do other country pairings (table 2). Additionally, since fewer RER series were found to switch the other way (from linear to STAR dynamics), we conclude that RER adjustments between developed countries are better described as linear processes. The prevalence of these linear dynamics in industrialized economies may be the result of a different monetary policy framework that can help to pin down the path of both inflation and prices.

Finally, the results for the developed-developing country pairs fall somewhere in between the two cases described above.

\section{Conclusion}

The goal of this paper has been to study the performance of tests commonly used to capture nonlinearities present in real exchange rates dynamics. Ahmad, Lo, and Mykhaylova (2013) demonstrate that these tests can overestimate the incidence of nonlinearity due to misspecification of the data generating process behind real exchange rates. Based on this observation, we re-examine the ability of the Teräsvirta and Anderson (1992) test to correctly identify RER dynamics by using a twopronged approach. First, we compare the results of the original Teräsvirta and Anderson (1992) test with those of the modification proposed in Ahmad, Lo, and Mykhaylova (2013) by studying the dynamics of 1431 bilateral real exchange rates during the most recent floating period (1970-2017). We find that approximately one quarter of the bilateral real exchange rate series that were initially identified as nonlinear under the Teräsvirta and Anderson (1992) test switch to being linear under the modification proposed by Ahmad, Lo, and Mykhaylova (2013), where exogenous variables are 
added to the test. Second, we employ the Monte Carlo approach to study the relationship between test performance and the underlying RER (linear) data generating process. Our results indicate that while the original test's results are influenced by the parameter values governing the DGP, the modified test performs equally well or better than the original in all exercises considered in this paper.

Our key finding is that standard nonlinearity test results are not independent of the data generating process driving real exchange rates. To this end, it is critically important for empirical researchers to have a theoretical model in mind before proceeding with estimations; otherwise, they risk misinterpreting the influence of exogenous variables on the RER dynamics as evidence of nonlinear behavior.

To assist in this endeavor and based on the findings above, the next step would be to identify a small set of right-hand side observed variables, which when included in nonlinearity tests, can correct the omitted variables bias. The variables included should address structural explanations of factors that drive real exchange rates, including those from trade, technological advances, international borrowing and lending, and monetary policy. Only then would rejections of the modified linearity test truly indicate potential sources of nonlinearities in real exchange rates. This we leave to future work. 


\section{References}

[1] Ahmad, Y., Glosser, S., 2011. Searching for Nonlinearities in Real Exchange Rates. Applied Economics, 43, 1829 - 1845. First published in iFirst, May 2009, 1-17.

[2] Ahmad, Y., Lo, M., Mykhaylova, O., 2013. Causes of Nonlinearities in Low-Order Models of the Real Exchange Rate. Journal of International Economics, 91, 128-141.

[3] Ahmad, Y., Staveley-O'Carroll, O.M., 2017. Exploring international differences in inflation dynamics. Journal of International Money and Finance, 79, 115-135.

[4] Aisen, A., Veiga, J., 2006. Does political instability lead to higher inflation: a panel data analysis. Journal of Money, Credit and Banking, 38, 1379-1389.

[5] Aguiar, M., Gopinath, G., 2007. Emerging market business cycles: the cycle is the trend. Journal of Political Economy, 115, 69-102.

[6] Backus, D., Smith, G., 1993. Consumption and real exchange rates in dynamic economies with non-traded goods. Journal of International Economics, 35, 297-316.

[7] Baum, C., Barkoulas, J. T., Caglayan, M., 2001. Nonlinear adjustment to purchasing power parity in the post-Bretton Woods era. Journal of International Money and Finance, 20, 379399.

[8] Chen, S., Engel, C., 2005. Does 'aggregation bias' explain the PPP puzzle? Pacific Economic Review, 10, 49-72.

[9] Davies, R.B., 1977. Hypothesis testing when a nuisance parameter is present only under the alternative. Biometrika, 64, 247-254. 
[10] Duarte, M., Restuccia, D., Waddle, A., 2007. Exchange rates and business cycles across countries. Economic Quarterly, Federal Reserve Bank of Richmond, 93, 57-76.

[11] Elbadawi, I., Soto, R., 2008. Theory and empirics of real exchange rates in developing countries. Working paper IE-PUC No. 337.

[12] Engel, C., Mark, N., West, K., 2007. Exchange Rate Models Are Not as Bad as You Think. NBER Macroeconomics Annual 200\%, 381-441.

[13] Ganguly, S., Breuer J., 2010. Nominal exchange rate volatility, relative price volatility, and the real exchange rate. Journal of International Money and Finance, 29, 840-856.

[14] Hausmann, R., Panizza, U., Rigobon, R., 2006. The long-run volatility puzzle of the real exchange rate. Journal of International Money and Finance, 25, 93-124.

[15] Kamps, C., 2006. New Estimates of Government Net Capital Stocks for 22 OECD Countries, 1960-2001. IMF Staff Papers, 53(1).

[16] Kilian, L., Taylor, M., 2003. Why is it So Difficult to Beat the Random Walk Forecast of Exchange Rates? Journal of International Economics, 60, 85-107.

[17] Lo, M., 2008. Nonlinear PPP deviations: a Monte Carlo investigation of their unconditional half-life. Studies in Nonlinear Dynamics and Econometrics, 12, Article 5.

[18] Michael, P., Nobay, R., Peel, D., 1997. Transaction Costs And Nonlinear Adjustments In Real Exchange Rates: An Empirical Investigation. Journal Of Political Economy, 105, 862-79.

[19] Obstfeld, M., Taylor, A., 1997. Nonlinear Aspects of Goods-Market Arbitrage and Adjustment: Heckscher's Commodity Points Revisited. Journal of Japanese and International Economics, $11,441-479$. 
[20] Paya, I., Peel, D., 2006. A new analysis of the determinants of the real dollar-sterling exchange rate: 1871 - 1994. Journal of Money, Credit and Banking, 38, 1971-1990.

[21] Reitz, S., Taylor, M., 2008. The Coordination Channel of Foreign Exchange Intervention: A Nonlinear Microstructural Analysis. European Economic Review, 52, 55-76.

[22] Rogoff, K., 1996. The Purchasing Power Parity Puzzle. Journal of Economic Literature, 34, 647-668.

[23] Sarantis, N., 1999. Modeling non-linearities in real effective exchange rates. Journal of International Money and Finance, 18, 27-45.

[24] Sarno, L., Taylor, M., 2001. Official intervention in the foreign exchange market: Is it effective and, if so, how does it work? Journal of Economic Literature, 39, 839-868.

[25] Taylor, A., Taylor, M., 2004. The Purchasing Power Parity Debate. Journal of Economic Perspectives, 18, 135-158.

[26] Taylor, M., 2006. Real Exchange Rates and Purchasing Power Parity: Mean-Reversion in Economic Thought. Applied Financial Economics, 16, 1-17.

[27] Taylor, M., Peel, D., Sarno, L., 2001. Nonlinear mean reversion in real exchange rates: towards a solution to the purchasing power parity puzzles, International Economic Review, 42, 10151042.

[28] Teräsvirta, T., 1994. Specification, Estimation, and Evaluation of Smooth Transition Autoregressive Models. Journal of American Statistics Association, 89, 208-218.

[29] Teräsvirta, T., Anderson, H., 1992. Characterizing Nonlinearities in Business Cycles Using Smooth Transition Autoregressive Models. Journal of Applied Econometrics, 7(S), S119-36. 
[30] Tsay, R., 1989. Testing and Modeling Threshold Autoregressive Processes. Journal of the American Statistical Association, 84, 231-240.

\section{A Data Sources and Description}

Sample countries (56): Argentina, Australia, Austria, Belgium, Bolivia, Botswana, Brazil, Canada, Colombia, Croatia, Cyprus, Czech Republic, Denmark, Ecuador, Estonia, Finland, France, Georgia, Germany, Hong Kong, Hungary, Indonesia, Ireland, Israel, Italy, Japan, Korea, Latvia, Lithuania, Luxembourg, Malaysia, Malta, Mauritius, Mexico, Netherlands, New Zealand, Norway, Peru, Philippines, Poland, Portugal, Romania, Russian Federation, Slovak Republic, Slovenia, South Africa, Spain, Sweden, Switzerland, Thailand, Turkey, Ukraine, United Kingdom, United States. The 17 countries in italics are included in our Eurozone subsample; the two notable omissions are Greece (also excluded from the full sample due to data limitations) and Lithuania (since it joined the Eurozone in 2015, close to the end of our time range).

Unless otherwise indicated, all data are taken from IMF's International Financial Statistics database and refer to the 1970Q1-2017Q4 period (although individual coverage varies by country).

$S_{t}^{i, U S D}$ : U.S. dollar per national currency, period average, EDNA.

$P_{t}^{i}$ : Consumer prices, all items, PCPI.

$Q_{t}^{i, j}$ : real exchange rate for a country pair $(i, j)$, calculated as

$$
Q_{t}^{i, j} \equiv \frac{S_{t}^{j, U S D} P_{t}^{j}}{S_{t}^{i, U S D} P_{t}^{i}}
$$


$\pi_{t}^{i}$ : CPI inflation, calculated as $\pi_{t}^{i} \equiv \log \left(\frac{P_{t}^{i}}{P_{t-1}^{i}}\right)$.

$Y_{t}^{i}$ : Gross domestic product, real, NGDP_R.

$I_{t}^{i}$ : Gross fixed capital formation, nominal, NFI.

$K_{t}^{i}$ : KTV: total net capital stock, volume, beginning-of-year stock. Source: Kamps (2006), annual data for the 1960-2002 period.

\section{A.1 Capital Stocks}

The available data on quarterly capital stocks is very limited in both country and time coverage. We therefore combine two data sets-IFS quarterly time series on investment $I_{t}^{i}$, and annual capital stock series $K_{t}^{i}$ from Kamps (2006) - to construct our own measure of this variable. Below we briefly outline the steps used in our computations.

We assume that each country's capital stock evolves according to the standard transition equation

$$
K_{t}^{i}=(1-\delta) K_{t-1}^{i}+I_{t}^{i}
$$

and that the depreciation rate $\delta$ is constant across time and countries. The latter is computed for each of the 11 non-Eurozone countries in the Kamps (2006) as follows: given the starting capital stock $K_{1970 Q 1}^{i}$ for $i=1, \ldots 11$, we calculate the depreciation rate $\hat{\delta}^{i}$ such that, iterating on $K_{1970 Q 1}^{i}$ using the above transition equation and the quarterly IFS data on investment, the computed $\hat{K}_{2002 Q 1}^{i}$ is approximately equal to the actual value reported in Kamps (2006). The average annualized value of $\hat{\delta}$ across the eleven economies is 0.1 .

We next compute the 1970Q1 average investment-capital stock ratio for all 22 economies in the 
Kamps (2006) dataset, which is equal to $\kappa \equiv 0.08$. W then set $K_{1970 Q 1}^{j}=\frac{I_{1970 Q 1}^{j}}{\kappa}$ for the rest of the countries in the IFS dataset not covered by Kamps (2006) calculations and iterate on it using (11) and the value of $\hat{\delta}$ obtained previously.

Since we are primarily concerned with business cycle fluctuations, we use logged variables in our nonlinearity tests. Therefore, the levels of the constructed capital stock series and subsequently our test results are independent of the above simplifying assumptions (about the starting values of investment-capital stock ratio and the rate of depreciation). 


\begin{tabular}{|lcccc|}
\hline \hline & \multicolumn{2}{c}{ Terasvirta-Anderson } & \multicolumn{2}{c|}{ Ahmad-Lo-Mykhaylova } \\
Specification & Number & Percent & Number & Percent \\
\hline Linear & 921 & 64.4 & 930 & 65.0 \\
LSTAR & 283 & 19.8 & 339 & 23.7 \\
ESTAR & 227 & 15.9 & 162 & 11.3 \\
Nonlinear Total & 510 & 35.6 & 501 & 35.0 \\
\hline Total & 1431 & 100.00 & 1431 & 100.00 \\
\hline
\end{tabular}

Table 1: Summary of nonlinearity tests in the 1970Q1-2017Q4 RER series. The percent values in each column are with respect to the total number of series indicated at the bottom of each column. 


\begin{tabular}{|c|c|c|c|c|c|c|c|c|}
\hline \multicolumn{9}{|c|}{ Panel A: Moving from TA to ALM } \\
\hline & \multicolumn{2}{|c|}{ Full Sample } & \multicolumn{2}{|c|}{ Industrial Pairs } & \multicolumn{2}{|c|}{ Developing Pairs } & \multicolumn{2}{|c|}{ Industrial-Developing Pairs } \\
\hline & Number & Percent & Number & Percent & Number & Percent & Number & Percent \\
\hline Linear/Linear & 809 & 56.5 & 278 & 63.9 & 132 & 47.8 & 399 & 55.4 \\
\hline$S T A R / S T A R$ & 389 & 27.2 & 81 & 18.6 & 99 & 35.9 & 209 & 29.0 \\
\hline Linear/STAR & 112 & 7.8 & 33 & 7.6 & 26 & 9.4 & 53 & 7.4 \\
\hline STAR/Linear & 121 & 8.5 & 43 & 9.9 & 19 & 6.9 & 59 & 8.2 \\
\hline Total & 1431 & 100.0 & 435 & 100.0 & 276 & 100.0 & 720 & 100.0 \\
\hline $\mathrm{n}$ & 54 & & 30 & & 24 & & & \\
\hline \multicolumn{9}{|l|}{ Proportion of: } \\
\hline Linear became STAR & & 12.2 & & 10.6 & & 16.5 & & 11.7 \\
\hline STAR became linear & & 23.7 & & 34.7 & & 16.1 & & 22.0 \\
\hline \multicolumn{9}{|c|}{ Panel B: Breakdowns of Switches } \\
\hline \multicolumn{9}{|c|}{ STAR/STAR } \\
\hline - LSTAR/LSTAR & 203 & 14.2 & 40 & 9.2 & 47 & 17.0 & 116 & 16.1 \\
\hline - LSTAR/ESTAR & 13 & 0.9 & 4 & 0.9 & 4 & 1.4 & 5 & 0.7 \\
\hline - ESTAR/LSTAR & 63 & 4.4 & 14 & 3.2 & 21 & 7.6 & 28 & 3.9 \\
\hline - ESTAR/ESTAR & 110 & 7.7 & 23 & 5.3 & 27 & 9.8 & 60 & 8.3 \\
\hline \multicolumn{9}{|l|}{ Linear $/ S T A R$} \\
\hline - Linear/LSTAR & 73 & 5.1 & 18 & 4.1 & 22 & 8.0 & 33 & 4.6 \\
\hline - Linear/ESTAR & 39 & 2.7 & 15 & 3.4 & 4 & 1.4 & 20 & 2.8 \\
\hline \multicolumn{9}{|l|}{ STAR/Linear } \\
\hline - LSTAR/Linear & 67 & 4.7 & 30 & 6.9 & 10 & 3.6 & 27 & 3.8 \\
\hline - ESTAR/Linear & 54 & 3.8 & 13 & 3.0 & 9 & 3.3 & 32 & 4.4 \\
\hline
\end{tabular}

Table 2: Incidence of nonlinearity in the 1970Q1-2017Q4 RER series. Panels A and B report the results from the comparison of the Teräsvirta-Anderson (TA; 1992) test and the Ahmad, Lo and Mykhaylova (ALM; 2013) comparison.

Notes: (i) Row one (two) in Panel A show the numbers of series that are found to be linear (nonlinear) using both the TA and the ALM tests, respectively. Rows three and four indicate the number of series that switched from being linear to nonlinear (and vice versa) when exogenous variables are included using the ALM test relative to the benchmark TA test.

(ii) The percent values in each column are with respect to the total number of series indicated at the bottom of each column.

(iii) The last two rows in Panel A report the fraction of all linear series under the TA test that became nonlinear after the inclusion of the exogenous variables using the ALM test, and vice versa. (iv) Panel B provides a breakdown of the switches in the results between the TA and ALM tests. 


\begin{tabular}{|lcccccc|}
\hline \hline & \multicolumn{2}{c}{ Eurozone (17) } & \multicolumn{2}{c|}{ Non-Eurozone (37) } & \multicolumn{2}{c|}{ Cross-Member Pairs } \\
& Number & Percent & Number & Percent & Number & Percent \\
\hline Linear/Linear & 79 & 58.1 & 341 & 51.2 & 389 & 62.0 \\
STAR/STAR & 25 & 18.4 & 220 & 33.0 & 142 & 22.6 \\
Linear/STAR & 15 & 11.0 & 54 & 8.1 & 43 & 6.9 \\
STAR/Linear & 17 & 12.5 & 51 & 7.7 & 53 & 8.5 \\
\hline Total & 136 & 100.0 & 666 & 100.0 & 627 & 100.0 \\
\hline Linear became STAR & & 16.0 & & 13.7 & & 10.0 \\
STAR became linear & & 40.5 & & 18.8 & & 27.2 \\
\hline
\end{tabular}

Table 3: Incidence of nonlinearity in the 1970Q1-2017Q4 RER series. Row one (two) shows the numbers of series that are found to be linear (nonlinear) using both the TA and the ALM tests, respectively. Rows three and four indicate the number of series that switched from being linear to nonlinear (and vice versa) when exogenous variables are included using the ALM test relative to the benchmark TA test. The last two rows report the fraction of all linear series under the TA test that became nonlinear after the inclusion of the exogenous variables using the ALM test, and vice versa.

\begin{tabular}{|c|c|}
\hline Original TA test & Modified ALM test \\
\hline $\begin{array}{cc}\text { Panel A: } & \beta_{q y, 1}=0.4, \beta_{q y, 2}= \\
d=1 & 15.08 \% \\
d=2 & 15.41 \%\end{array}$ & $\begin{array}{l}5.20 \% \\
5.16 \%\end{array}$ \\
\hline $\begin{array}{cc}\text { Panel B: } & \beta_{q y, 1}=\beta_{q y, 2}=0 \\
d=1 & 4.05 \% \\
d=2 & 4.17 \%\end{array}$ & $\begin{array}{l}4.03 \% \\
4.43 \%\end{array}$ \\
\hline
\end{tabular}

Table 4: Rejection rates of the null hypothesis (7). The calculations are based on 10,000 simulations, each 450 periods long, of the real exchange rate series given by (10). 